\title{
BIHOLOMORPHIC MAPS BETWEEN TEICHMÜLLER SPACES
}

\author{
VLADIMIR MARKOVIC
}

\begin{abstract}
In this paper we study biholomorphic maps between Teichmüller spaces and the induced linear isometries between the corresponding tangent spaces. The first main result in this paper is the following classification theorem. If $M$ and $N$ are two Riemann surfaces that are not of exceptional type, and if there exists a biholomorphic map between the corresponding Teichmüller spaces Teich $(M)$ and $\operatorname{Teich}(N)$, then $M$ and $N$ are quasiconformally related. Also, every such biholomorphic map is geometric. In particular, we have that every automorphism of the Teichmüller space Teich $(M)$ must be geometric. This result generalizes the previously known results (see [2], [5], [7]) and enables us to prove the well-known conjecture that states that the group of automorphisms of Teich $(M)$ is isomorphic to the mapping class group of $M$ whenever the surface $M$ is not of exceptional type. In order to prove the above results, we develop a method for studying linear isometries between $L^{1}$-type spaces. Our focus is on studying linear isometries between Banach spaces of integrable holomorphic quadratic differentials, which are supported on Riemann surfaces. Our main result in this direction (Theorem 1.1) states that if $M$ and $N$ are Riemann surfaces of nonexceptional type, then every linear isometry between $A^{1}(M)$ and $A^{1}(N)$ is geometric. That is, every such isometry is induced by a conformal map between $M$ and $N$.
\end{abstract}

\section{Introduction}

In this paper, unless it is specified otherwise, every Riemann surface is assumed to carry the unique complete metric of curvature -1 , which we call the hyperbolic metric. If $M$ and $N$ are a pair of Riemann surfaces, by $z=x+i y$ and $z_{1}=x_{1}+i y_{1}$ we denote local parameters on $M$ and $N$, respectively.

Let $M$ be a Riemann surface, and let $A^{1}(M)$ denote the Banach space of all integrable holomorphic quadratic differentials $\phi(d z)^{2}=\phi$ with the Banach norm

$$
\|\phi\|_{1}=\int_{M}|\phi| d x d y .
$$

DUKE MATHEMATICAL JOURNAL

Vol. 120, No. 2, (C) 2003

Received 17 May 2002. Revision received 17 January 2003.

2000 Mathematics Subject Classification. Primary 30F60; Secondary 30F20.

Author's research partly supported by an Engineering and Physical Sciences Research Council Advanced Research Fellowship.

405 
This space is also sometimes called the Bergman space $A^{1}(M)$. Let $N$ be another Riemann surface, and denote by $A^{1}(N)$ the corresponding Bergman space. One of the main goals of this paper is to characterize surjective linear isometries between the Banach spaces $A^{1}(M)$ and $A^{1}(N)$.

\section{Definition}

A surjective linear isometry $T: A^{1}(M) \rightarrow A^{1}(N)$ is said to be geometric if there exist a conformal map $\alpha: M \rightarrow N$ and a complex number $\theta,|\theta|=1$, such that

$$
T^{-1}(\phi)=\theta(\phi \circ \alpha)\left(\alpha^{\prime}\right)^{2}
$$

for every $\phi \in A^{1}(N)$.

The following problem has been extensively studied (see [7], [3], [2], [5]).

\section{Problem 1.1}

Let $M$ and $N$ be two Riemann surfaces that are not of exceptional type. Then every surjective linear isometry $T: A^{1}(M) \rightarrow A^{1}(N)$ is geometric.

We also mention the following special case of Problem 1.1, which was introduced in [2].

\section{Problem 1.2}

Let $M_{1}$ and $M_{2}$ be two Riemann surfaces quasiconformally related to a Riemann surface $M$, and assume that $M$ is not of exceptional type. Then, every surjective linear isometry $T: A^{1}\left(M_{1}\right) \rightarrow A^{1}\left(M_{2}\right)$ is geometric.

Remark. A Riemann surface $M$ is said to be of exceptional type if it is of finite hyperbolic area and $2 g+n \leq 4$, where $g$ is the genus of $M$ and $n$ is the number of points in the boundary of $M$. Also, we say that two Riemann surfaces are conformally or quasiconformally related if there exists a conformal or quasiconformal map between them. If Problem 1.2 has a positive answer for $M$, we say that $M$ has the isometry property.

It has been known since H. Royden [7] (see also [4]) that if $M$ and $N$ are closed Riemann surfaces (which are not of exceptional type), then all linear isometries between $A^{1}(M)$ and $A^{1}(N)$ are geometric. This result was extended in [3] to the case of all Riemann surfaces of finite hyperbolic area, that is, all Riemann surfaces where the dimension of the space $A^{1}(M)$ is finite. In recent years, Problem 1.1 has been solved in many important cases. In [2] it has been proved that Problem 1.1 has a positive solution if $M$ and $N$ are of finite topological type (finitely generated fundamental group). 
In [5] it was proved that Problem 1.1 has a positive solution for all surfaces of finite genus. In this paper we settle the general case.

\section{THEOREM 1.1}

Suppose that Riemann surfaces $M$ and $N$ are of nonexceptional type. Let $T$ : $A^{1}(M) \rightarrow A^{1}(N)$ be a surjective linear isometry. Then the isometry $T$ is geometric. The surfaces $M$ and $N$ are conformally related and therefore homeomorphic.

Remark. Note that in the above theorem we do not assume that $M$ and $N$ are homeomorphic. A Riemann surface $M$ is said to have finite analytical type if it can be obtained from a closed surface by deleting at most finitely many points. Therefore we derive a positive solution to Problem 1.1; that is, every Riemann surface that is not of exceptional type has the isometry property. Clearly, the positive solution to Problem 1.1 implies that Problem 1.2 has a positive solution as well.

Problem 1.1 is interesting in its own right. However, the importance of this problem also comes from Teichmüller theory. Denote by Teich $(M)$ the Teichmüller space of $M$. Let $N$ be another Riemann surface. One of the central questions in Teichmüller theory is how to describe all biholomorphic maps between $\operatorname{Teich}(M)$ and $\operatorname{Teich}(N)$. In particular, it is important to describe biholomorphic maps of Teich $(M)$ onto itself. These maps are called automorphisms of Teich $(M)$. They form the group called $\operatorname{Aut}(\operatorname{Teich}(M))$.

Every quasiconformal map $g: M \rightarrow N$ induces a biholomorphic map $\rho_{g}$ : $\operatorname{Teich}(M) \rightarrow \operatorname{Teich}(N)$. This map is called geometric (see [2] for the definition of the notion of geometric maps between Teichmüller spaces). We define the mapping class group $\mathrm{MC}(M)$ to be the group of all classes of quasiconformal maps $g: M \rightarrow M$. Every element $g$ of the group $\mathrm{MC}(M)$ induces the automorphism $\rho_{g}$ of Teich $(M)$. The following conjecture is well

\section{CONJECTURE 1.1}

If the Riemann surface $M$ is not of exceptional type, then

$$
\operatorname{Aut}(\operatorname{Teich}(M))=\operatorname{MC}(M)
$$

In his celebrated paper [7], Royden showed that in the case when $M$ and $N$ are closed surfaces of finite genus (and not of exceptional type), then every biholomorphic map $F: \operatorname{Teich}(M) \rightarrow \operatorname{Teich}(N)$ is geometric. In particular, every automorphism of $\operatorname{Teich}(M)$ is geometric and is represented by an element of $\operatorname{MC}(M)$. This is a very important result in the theory of finitely dimensional Teichmüller spaces. Since then, the 
question of extending this theorem to other Riemann surfaces, and their Teichmüller spaces, has been studied in [2], [3], [5], and [7].

Remark. Royden's result about automorphisms of Teichmüller spaces was extended to Riemann surfaces of finite genus in [2] and [5] by solving Problem 1.1 in these cases. A Riemann surface is of finite genus if there exists a closed Riemann surface $M^{*}$ such that $M$ can be conformally embedded in $M^{*}$.

Our next theorem settles this conjecture.

THEOREM 1.2

Suppose that $M$ is a Riemann surface that is not of exceptional type. Then

$$
\operatorname{Aut}(\operatorname{Teich}(M))=\operatorname{MC}(M)
$$

If Riemann surfaces $M$ and $N$ are not quasiconformally related, one still can ask if there exists a biholomorphic map $F: \operatorname{Teich}(M) \rightarrow \operatorname{Teich}(N)$. The following classification theorem is a corollary of Theorem 1.1 and some important results from [2] (see Section 9 for details).

\section{THEOREM 1.3}

Suppose that Riemann surfaces $M$ and $N$ are not of exceptional type, and let $F$ : $\operatorname{Teich}(M) \rightarrow \operatorname{Teich}(N)$ be a biholomorphic map. Then, we have that $M$ and $N$ are quasiconformally related, and the map $F$ is geometric.

The method we develop to prove Theorem 1.1 is different from the methods used in [2], [5], and [7]. Although in this paper we are focused on linear isometries between $L^{1}$ Bergman spaces of quadratic differentials on Riemann surfaces, parts of the proof of Theorem 1.1 could be used in studying linear isometries between subspaces of general $L^{p}$-type spaces (here $p>0$, and $p$ is not an even integer). We now list the main steps in the proof of Theorem 1.1.

Theorem 2.1 is the first crucial step toward the proof of Theorem 1.1. It is particularly interesting because it holds in the context of arbitrary $L^{1}$-spaces. In Theorem 3.3 we prove an important separation property of the Bergman spaces $A^{1}(M)$, where $M$ is of nonexceptional type. In a way, Theorem 3.3 states that the space $A^{1}(M)$ contains enough elements to completely determine the underlying Riemann surface $M$.

In Section 4, as a direct corollary of Theorems 2.1 and 3.3, we construct the map $\beta$ with the following properties. The map $\beta$ is defined almost everywhere on $M$, and it is a bijection onto some set of the full measure in $N$. In particular, $\beta$ is measurepreserving; that is, the following holds. Let $F$ be a measurable subset of $M$. Then for 
every $\phi \in A^{1}(M)$, we have

$$
\int_{F}|\phi| d x d y=\int_{\beta(F)}|T(\phi)| d x_{1} d y_{1} .
$$

The identity (1.1) shows that the map $\beta$ is the map we could use to prove Theorem 1.1. The main obstacle is that we do not know whether $\beta$ is defined everywhere on $M$ and, in particular, whether $\beta$ is conformal. In Sections $5-8$, we show that there exists a conformal map $\alpha: M \rightarrow N$ such that $\alpha=\beta$ almost everywhere on $M$. With this we conclude the proof of Theorem 1.1. In Section 9, we give the proofs of Theorems 1.2 and 1.3 .

\section{Preliminary results}

In the following theorem the measures $\mu$ and $v$ are finite and positive measures on sets $X$ and $Y$, respectively. The nature of these sets plays no role in what follows, so we do not specify them. The following result was proved in [8].

\section{THEOREM 2.1}

Let $f_{1}, \ldots, f_{n} \in L^{1}(\mu)$ and $g_{1}, \ldots, g_{n} \in L^{1}(v)$ be such that for every $n$-tuple of complex numbers $\alpha_{k}, k=1,2, \ldots, n$, we have

$$
\int\left|1+\sum_{k=1}^{n} \alpha_{k} f_{k}\right| d \mu=\int\left|1+\sum_{k=1}^{n} \alpha_{k} g_{k}\right| d \nu .
$$

Then, for every bounded Borel measurable function (and for every real-valued nonnegative Borel function) $u: \mathbf{C}^{n} \rightarrow \mathbf{C}$, we have

$$
\int u\left(f_{1}, \ldots, f_{n}\right) d \mu=\int u\left(g_{1}, \ldots, g_{n}\right) d \nu .
$$

Furthermore, let $I: X \rightarrow \mathbf{C}^{n}$ and $J: Y \rightarrow \mathbf{C}^{n}$ be the maps $I=\left(f_{1}, \ldots, f_{n}\right)$ and $J=\left(g_{1}, \ldots, g_{n}\right)$, respectively. Then we have

$$
\mu\left(I^{-1}(E)\right)=v\left(J^{-1}(E)\right)
$$

for every Borel set $E$ in $\mathbf{C}^{n}$.

Remark. In W. Rudin's paper [8], Theorem 2.1 was proved in a more general context. In fact, it holds if instead of $L^{1}(\mu)$ we consider $L^{p}(\mu)$, where $p>0$ and $p$ is not an even integer.

The following two lemma are easy corollaries of Theorem 2.1. 
LEMMA 2.1

Suppose that $\phi_{k}, k=0,1,2, \ldots, n, n \in \mathbf{N}$, are elements of $A^{1}(M)$, and suppose that $\psi_{k}, k=0,1,2, \ldots, n$, are elements of $A^{1}(N)$, such that for every $n$-tuple of complex numbers $\alpha_{k}, k=1,2, \ldots, n$, we have

$$
\left\|\phi_{0}+\sum_{k=1}^{n} \alpha_{k} \phi_{k}\right\|_{1}=\left\|\psi_{0}+\sum_{k=1}^{n} \alpha_{k} \psi_{k}\right\|_{1} .
$$

If neither $\phi_{0}$ nor $\psi_{0}$ is constantly zero, then for every real-valued nonnegative Borel function $u: \mathbf{C}^{n} \rightarrow \mathbf{C}$ we have

$$
\int_{M} u\left(\frac{\phi_{1}}{\phi_{0}}, \ldots, \frac{\phi_{n}}{\phi_{0}}\right)\left|\phi_{0}\right| d x d y=\int_{N} u\left(\frac{\psi_{1}}{\psi_{0}}, \ldots, \frac{\psi_{n}}{\psi_{0}}\right)\left|\psi_{0}\right| d x_{1} d y_{1} .
$$

Proof

Set $d \mu=\left|\phi_{0}\right| d x d y$ and $d v=\left|\psi_{0}\right| d x_{1} d y_{1}$, where $z=x+i y$ and $z_{1}=x_{1}+i y_{1}$ are local parameters on $M$ and $N$, respectively. The measures $\mu$ and $v$ are well defined on $M$ and $N$, respectively. The equality (2.3) can be written as

$$
\int_{M}\left|1+\sum_{k=1}^{n} \alpha_{k} \frac{\phi_{k}}{\phi_{0}}\right| d \mu=\int_{N}\left|1+\sum_{k=1}^{n} \alpha_{k} \frac{\psi_{k}}{\psi_{0}}\right| d \nu .
$$

The proof now follows from Theorem 2.1.

By the Lebesgue measure $\mathbf{m}_{M}$ on a Riemann surface $M$, we consider the measure that has the following properties. The universal covering surface of $M$ is the unit disc. Let $F$ be a subset of $M$ which is contained in an injectively embedded hyperbolic disc on $M$. Then we say that $F$ is Lebesgue measurable on $M$ if the set in the unit disc, which represents a lift of $F$, is Lebesgue measurable as a subset of the complex plane. The measure of the set $F$ is equal to its hyperbolic area. Note that the measure $\mu$ introduced above has the same families of measurable sets, and also the same families of sets of measure zero, as the measure $\mathbf{m}_{M}$. Since these are the only two types of measures on Riemann surfaces we deal with in the remainder of this paper, we use terms like measurable set, set of measure zero, set of positive measure, and so on, without specifying the measure.

Set

$$
I(p)=\left(\frac{\phi_{1}}{\phi_{0}}(p), \ldots, \frac{\phi_{n}}{\phi_{0}}(p)\right)
$$

for $p \in M$, and set

$$
J(q)=\left(\frac{\psi_{1}}{\psi_{0}}(q), \ldots, \frac{\psi_{n}}{\psi_{0}}(q)\right)
$$

for $q \in N$. We have the following. 
LEMMA 2.2

Under the same set of assumptions as in the previous lemma, we have that the following holds. Let $F$ be a compact set in $M$ such that $\phi_{0}$ has no zeros in $F$. Let $u: \mathbf{C}^{n} \rightarrow \mathbf{C}$ be the function defined to be 1 on the set $I(F)$ and to be equal to zero elsewhere in $\mathbf{C}^{n}$. Then we have

$$
\int_{M}(u \circ I) d \mu=\int_{I^{-1}(I(F))} d \mu=\int_{J^{-1}(I(F))} d v=\int_{N}(u \circ J) d v .
$$

Remark. The set $I^{-1}(I(F))$ is the set of all points on $M$ which are mapped in the set $I(F)$ by the map $I$. Clearly, $F$ is contained in $I^{-1}(I(F))$. Also, the set $J^{-1}(I(F))$ is the set of all points on $N$ which are mapped in the set $I(F)$ by the map $J$. However, in general $J^{-1}(I(F))$ may be empty as well, and it is a corollary of Lemma 2.2 that $J^{-1}(I(F))$ has positive measure in $N$ whenever $F$ has positive measure in $M$.

Proof

Since the map $I$ is continuous, the set $I(F)$ is a compact set in $\mathbf{C}^{n}$. This implies that the function $u$ is a Borel function. The proof of Lemma 2.2 now follows from Lemma 2.1.

\section{Separation properties of the space $A^{1}(M)$}

Let $S_{M}$ be the subset of a Riemann surface $M$ defined as follows.

\section{Definition}

We say that $p_{0} \in S_{M}$ if for every point $p \in M, p \neq p_{0}$, there exists a differential $\phi \in A^{1}(M)$ such that $\phi(p) \phi\left(p_{0}\right)=0$ and such that $\phi(p)$ and $\phi\left(p_{0}\right)$ are not both equal to zero.

Although $\phi \in A^{1}(M)$ is not a function on $M$, the statements $\phi(p)=0$ and $\phi(p) \neq 0$ are well defined, and so the above definition is independent of the choice of a local parameter.

Denote by $\Gamma(M)$ the Hilbert space of all integrable holomorphic $(1,0)$ forms on $M$. $\Gamma(M)$ is also known as the space of all Abelian square-integrable forms on $M$. The Hilbert norm of $\varphi d z=\varphi, \varphi \in \Gamma(M)$, is given by

$$
\|\varphi\|_{2}=\left(\int_{M}|\varphi|^{2}\right)^{1 / 2}=\left\|\varphi^{2}\right\|_{1}^{1 / 2}
$$

\section{THEOREM 3.1}

Suppose that the dimension of the space $\Gamma(M)$ is at least 3. Then the set $M \backslash S_{M}$ is a discrete set of points in $M$. Furthermore, if $p^{\prime}$ is a puncture in the boundary of $M$, then $p^{\prime}$ is not a cluster point of the set $M \backslash S_{M}$. 
Remark. By saying that $p^{\prime}$ is a puncture in the boundary of $M$, we mean that there exists another Riemann surface $\tilde{M}$ such that the surface $M$ is obtained from $\tilde{M}$ by deleting the point $p^{\prime}$. The assumption that the dimension of the space $\Gamma(M)$ is at least 3 is satisfied if the genus of the surface $M$ is infinite. The assumption is also satisfied by many surfaces of finite genus, but an even sharper theorem holds for such surfaces (see Theorem 3.2).

Proof

We say that a point $p_{0} \in D_{M}$ if $p_{0} \in M$ and if there exists $\phi_{0} \in \Gamma(M)$ with a zero of order 2 at $p_{0}$; that is,

$$
\phi_{0}\left(z-z_{0}\right)=A_{0}\left(z-z_{0}\right)^{2}+o\left(\left(z-z_{0}\right)^{2}\right), \quad A_{0} \neq 0,
$$

where $z$ is close to $z_{0}$. Here $z(p)=z$ is a local parameter near $p_{0}$, and $z\left(p_{0}\right)=z_{0}$.

We first show that $D_{M}$ is contained in $S_{M}$. Let $p_{0} \in M$. Denote by $\varphi_{0}$ the adjoint kernel differential with the reference point $p_{0}$. It is well known (see [1] and [10, page 43]) that this differential exists for every Riemann surface (not necessarily hyperbolic). It has the following properties. The kernel $\varphi_{0}$ is a $(1,0)$ meromorphic form on $M$, with the second-order pole at $p_{0}$. Furthermore, if $U$ denotes an arbitrary open set containing $p_{0}$, then

$$
\int_{M \backslash U}\left|\varphi_{0}\right|^{2}<\infty
$$

In particular, (3.2) implies that $p_{0}$ is the only pole of the differential $\varphi_{0}$.

Remark. The existence of the above differential was proved in [1] (also see [10]). For example, if $M$ is the complex plane, and $p_{0}=0$, then the differential $\varphi_{0}=1 / z^{2}$. The kernel $\varphi_{0}$ clearly has the second-order pole at zero, and $\left(\varphi_{0}\right)^{2}$ is integrable on the sphere minus some open set containing zero. In particular, if the space $\Gamma(M)$ is not trivial, then the the kernel differential $\varphi_{0}$ is characterized as the unique (up to the multiplication by a nonzero constant) differential with the above properties and which annihilates the space $\Gamma(M)$ in the sense of the standard inner product (computed as a Cauchy principal value).

Now suppose that $p_{0} \in D_{M}$. Denote by $\phi_{0}$ the differential satisfying (3.1), and denote by $\varphi_{0}$ the kernel differential with the reference point $p_{0}$. Let $p \neq p_{0}$ be an arbitrary point in $M$. If $\phi_{0}(p) \neq 0$, and since $\phi_{0}^{2} \in A^{1}(M)$, we conclude that $p_{0} \in S_{M}$. If $\phi_{0}(p)=0$, then we have $\phi_{0}(p) \varphi_{0}(p)=0$. From (3.1) it follows that $\phi_{0}\left(p_{0}\right) \varphi_{0}\left(p_{0}\right) \neq$ 0 . Also, from (3.1) and (3.2) it follows that $\phi_{0} \varphi_{0} \in A^{1}(M)$. We conclude that $p_{0} \in S_{M}$ in this case too.

To finish the proof it is enough to show that $M \backslash D_{M}$ is a discrete set of points in $N$. Also, if $p^{\prime}$ is a puncture in the boundary of $M$, we have to show that $p^{\prime}$ is not 
a cluster point of the set $M \backslash D_{M}$. Since $\Gamma(M)$ is at least of the dimension 3, we can choose three linearly independent differentials from $\Gamma(M)$. Denote them by $\varphi, \phi, \psi$. We have that $\varphi, \phi$, and $\psi$ are holomorphic at every puncture in the boundary of $M$.

Let $p^{\prime}$ be a point of $M$ or a puncture on the boundary of $M$, and let $z$ be a local coordinate in a neighborhood $U$ of $p^{\prime}$. For $z \in U$, set

$$
\begin{aligned}
a(z) \varphi(z)+b(z) \phi(z)+c(z) \psi(z) & =0, \\
a(z) \varphi^{\prime}(z)+b(z) \phi^{\prime}(z)+c(z) \psi^{\prime}(z) & =0, \\
a(z) \varphi^{\prime \prime}(z)+b(z) \phi^{\prime \prime}(z)+c(z) \psi^{\prime \prime}(z) & =1 .
\end{aligned}
$$

This system of equations can be solved for $a(z), b(z), c(z)$ at every point $z$ where the Wronskian determinant of $\varphi, \phi, \psi$ is not zero. Since $\varphi, \phi, \psi$ are linearly independent, the set of zeros of the Wronskian determinant is a discrete set in $M$, and also, this set cannot accumulate at any puncture in the boundary of $M$.

Let $p \in M$ be a point where the system (3.3) has a solution. Then we have $p \in D_{M}$. This implies that the set $M \backslash D_{M}$ cannot accumulate at any point of $M$ (or a puncture in the boundary of $M$ ).

In the remainder of this section, we deal with the case of finite genus surfaces. The following lemma is well known. I thank the referee for pointing out this lemma and its proof to me.

LEMMA 3.1

Let $M^{*}$ be a compact Riemann surface of genus $g$, and let $D$ be a divisor of degree $n \geq 0$ on $M^{*}$. Suppose $2 g+n \geq 5$. Let $V$ be the vector space of meromorphic quadratic differentials $\varphi$ on $M^{*}$ such that $(\varphi)+D \geq 0$. (Here $(\varphi)$ denotes the divisor of $\varphi$.) If $p$ and $q$ are distinct points of $M^{*}$ which do not belong to the support of $D$, then there is a $\varphi$ in $V$ that is zero at $p$ but not at $q$.

Proof

Let $\omega$ be a nontrivial one-form on $M^{*}$. We have $\varphi \in V$ if and only if $\varphi=f \omega^{2}$, where $f$ is a meromorphic function on $M^{*}$ with $(f)+2(\omega)+D \geq 0$.

Let $E$ be the divisor $2(\omega)+D$. Then $V$ has dimension $\operatorname{dim}(E)$. Also, the space of $\varphi$ in $V$ such that $\varphi$ has a zero at $p$ has dimension $\operatorname{dim}(E-p)$, and the space of $\varphi$ in $V$ with zeros at both $p$ and $q$ has dimension $\operatorname{dim}(E-p-q)$.

By hypothesis, $2 g+n \geq 5$, so the divisors $(\omega)-E,(\omega)-E+p$, and $(\omega)-E+$ $p+q$ all have negative degree. Therefore the Riemann-Roch theorem implies that $\operatorname{dim}(V)=\operatorname{dim}(E)=3 q+n-3, \operatorname{dim}(E-p)=\operatorname{dim}(V)-1$, and $\operatorname{dim}(E-p-q)=$ $\operatorname{dim}(V)-2$. In particular, the space of $\varphi$ in $V$ with zeros at both $p$ and $q$ has lower dimension than the subspace with zeros at $p$. 
THEOREM 3.2

Suppose that $M$ has finite genus and is not of exceptional type. Then $M=S_{M}$

Remark. It is easy to verify that the above theorem does not hold for exceptional-type surfaces.

Proof

Let $M^{*}$ be the closed surface of the same genus as $M$, such that $M$ is obtained from $M^{*}$ by deleting points $p_{1}, \ldots, p_{k} \in M^{*}$. (The set of points $p_{1}, \ldots, p_{k}$ may be empty.) Define the divisor $D$ on $M^{*}$ by $D=p_{1}+\cdots+p_{k}$. The theorem now follows from Lemma 3.1.

Combining Theorems 3.1 and 3.2, we obtain the following theorem.

THEOREM 3.3

Suppose that $M$ is a Riemann surface of nonexceptional type. Then the set $M \backslash S_{M}$ is a discrete set of points in $M$. Furthermore, if $p^{\prime}$ is a puncture in the boundary of $M$, then $p^{\prime}$ is not a cluster point of the set $M \backslash S_{M}$.

\section{Construction of the map $\beta$, and its properties}

From now on, we assume that $M$ and $N$ are Riemann surfaces of nonexceptional type. We do not assume that $M$ and $N$ are homeomorphic.

Let $T: A^{1}(M) \rightarrow A^{1}(N)$ be a surjective linear isometry. Since $A^{1}(M)$ is a separable Banach space, we can choose a sequence of distinct differentials $\left(\phi_{k}\right), k=$ $0,1,2, \ldots$, such that for every $\phi \in A^{1}(M)$ there exists a subsequence of $\left(\phi_{k}\right)$, which is norm converging to $\phi$, in the norm on $A^{1}(M)$. In addition, we choose $\phi_{0}$ and $\phi_{1}$ to be linearly independent.

Denote by $M_{1}$ the set $S_{M}$ minus the two discrete sets of points. The first one is the set of points where $\phi_{0}$ has zeros, and the second is where the meromorphic function $\phi_{1} / \phi_{0}$ has the first derivative equal to zero. It follows from Theorem 3.3 that $M \backslash M_{1}$ is a discrete set of points in $M$. Note that if $p^{\prime}$ is a puncture in the boundary of $M$, then the function $\phi_{1} / \phi_{0}$ is a well-defined meromorphic function in a given neighborhood of $p^{\prime}$. Therefore the set of points where $\phi_{0}$ has zeros, and also the set of points where $\phi_{1} / \phi_{0}$ has the first derivative equal to zero, does not have the point $p^{\prime}$ as its cluster point. Since we proved in Section 3 that the set $S_{M}$ has the same property, we conclude that the set $M \backslash M_{1}$ does not have the point $p^{\prime}$ as its cluster point. We define the set $N_{1}$ in $N$ in the same way, using the sequence $T\left(\phi_{k}\right)$ in $A^{1}(N)$. The set $N \backslash N_{1}$ has the same discreteness properties as $M \backslash M_{1}$.

The notation and assumptions introduced above remain valid until the end of this 
paper. In this section we work with $M_{1}$ and $N_{1}$, and we return to $M$ and $N$ later.

For every $n \in \mathbf{N}$, we define the maps $I_{n}: M_{1} \rightarrow \mathbf{C}^{n}$ and $J_{n}: N_{1} \rightarrow \mathbf{C}^{n}$ by

$$
I_{n}(p)=\left(\frac{\phi_{1}}{\phi_{0}}(p), \ldots, \frac{\phi_{n}}{\phi_{0}}(p)\right)
$$

for $p \in M_{1}$ and

$$
J_{n}(q)=\left(\frac{T\left(\phi_{1}\right)}{T\left(\phi_{0}\right)}(q), \ldots, \frac{T\left(\phi_{n}\right)}{T\left(\phi_{0}\right)}(q)\right)
$$

for $q \in N_{1} . I_{n}$ and $J_{n}$ are holomorphic maps. In particular, from the definition of $M_{1}$ it follows that for every $p \in M_{1}$ there is a neighborhood $U$ of $p$, and a biholomorphic map $\tilde{I}_{n}: U \times \mathbf{C}^{n-1} \rightarrow \mathbf{C}^{n}$, such that $I_{n}(p)=\tilde{I}_{n}(p, 0)$. This implies that if $F$ is a Borel set on $M_{1}$, then $I_{n}(F)$ is a Borel set in $\mathbf{C}^{n}$. A similar observation holds for $N_{1}$.

Also, define the maps $I_{\infty}: M_{1} \rightarrow \mathbf{C}^{\mathbf{N}}$ and $J_{\infty}: N_{1} \rightarrow \mathbf{C}^{\mathbf{N}}$ as

$$
I_{\infty}(p)=\left(\frac{\phi_{1}}{\phi_{0}}(p), \ldots\right)
$$

for $p \in M_{1}$ and

$$
J_{\infty}(q)=\left(\frac{T\left(\phi_{1}\right)}{T\left(\phi_{0}\right)}(q), \ldots\right)
$$

for $q \in N_{1}$. The product topology of pointwise convergence makes the space $\mathbf{C}^{\mathbf{N}}$ a Fréchet space. We have that maps $I_{\infty}$ and $J_{\infty}$ are holomorphic, and they map Borel sets to Borel sets for the same reason that the maps $I_{n}$ and $J_{n}$ do.

Suppose that $z=x+i y$ and $z_{1}=x_{1}+i y_{1}$ are local parameters on $M$ and $N$, respectively. Set $d \mu=\left|\phi_{0}\right| d x d y$, and set $d v=\left|T\left(\phi_{0}\right)\right| d x_{1} d y_{1}$. The measures $\mu, v$ are well defined on $M$ and $N$, respectively.

LEMMA 4.1

Let $p \in M_{1}$, and let $q \in N_{1}$. Then

$$
\begin{aligned}
& \bigcap_{n=1}^{\infty} I_{n}^{-1}\left(I_{n}(p)\right)=\{p\}, \\
& \bigcap_{n=1}^{\infty} J_{n}^{-1}\left(J_{n}(q)\right)=\{q\} .
\end{aligned}
$$

In other words, the maps $I_{\infty}$ and $J_{\infty}$ are injective.

Proof

Suppose that there exists $p^{\prime} \in M, p \neq p^{\prime}$, such that $p^{\prime} \in \bigcap_{n=1}^{\infty} I_{n}^{-1}\left(I_{n}(p)\right)$. Since $\phi_{n}$ is dense in $A^{1}(M)$, we conclude that

$$
\frac{\phi}{\phi_{0}}(p)=\frac{\phi}{\phi_{0}}\left(p^{\prime}\right)
$$


for every $\phi \in A^{1}(M)$. From Theorem 3.3 we know that there exists $\psi \in A^{1}(M)$ such that either $\psi(p)=0$ and $\psi\left(p^{\prime}\right) \neq 0$ or $\psi(p) \neq 0$ and $\psi\left(p^{\prime}\right)=0$. But this contradicts (4.1). The proof for $N_{1}$ is similar.

Let $G_{M}$ be the set of all points $p \in M_{1}$ such that the set

$$
\bigcap_{n=1}^{\infty} J_{n}^{-1}\left(I_{n}(p)\right)
$$

is a nonempty subset of $N_{1}$. Also, let $G_{N}$ be the set of all points $q \in N_{1}$ such that the set

$$
\bigcap_{n=1}^{\infty} I_{n}^{-1}\left(J_{n}(q)\right)
$$

is a nonempty subset of $M_{1}$.

Observe that

$$
G_{M}=I_{\infty}^{-1}\left(J_{\infty}\left(N_{1}\right)\right), \quad G_{N}=J_{\infty}^{-1}\left(I_{\infty}\left(M_{1}\right)\right)
$$

and

$$
I_{\infty}\left(G_{M}\right)=J_{\infty}\left(G_{N}\right)=I_{\infty}\left(M_{1}\right) \cap J_{\infty}\left(N_{1}\right) .
$$

In particular, we have that $G_{M}$ and $G_{N}$ are Borel sets.

LEMMA 4.2

We have

$$
\mu\left(M_{1} \backslash G_{M}\right)=v\left(N_{1} \backslash G_{N}\right)=0
$$

Proof

Let $K_{1}$ be an arbitrary compact set in $N_{1}$. Suppose that there exists a compact set $F \subset M_{1}$ such that $\mu(F)>0$ and $F \cap G_{M}$ is an empty set. Note that $J_{n}^{-1}\left(I_{n}(F)\right)$ contains $J_{n+1}^{-1}\left(I_{n+1}(F)\right)$. Set

$$
A_{n}=v\left(J_{n}^{-1}\left(I_{n}(F)\right) \cap K_{1}\right) .
$$

From the assumptions on the set $F$, we find that the set

$$
\bigcap_{n=1}^{\infty} J_{n}^{-1}\left(I_{n}(F)\right)
$$

is an empty set. Therefore we have $A_{n} \rightarrow 0$ when $n \rightarrow \infty$. 
From Lemma 2.2 we have

$$
\mu(F) \leq \mu\left(I_{n}^{-1}\left(I_{n}(F)\right)\right)=\int_{I_{n}^{-1}\left(I_{n}(F)\right)} d \mu=\int_{J_{n}^{-1}\left(I_{n}(F)\right)} d v=v\left(J_{n}^{-1}\left(I_{n}(F)\right)\right) .
$$

The lim inf of the sequence of values of the integrals on the right-hand side in (4.2), when $n \rightarrow \infty$, is at most equal to $v\left(N_{1} \backslash K_{1}\right)$. By varying the set $K_{1}$, we obtain a contradiction.

Therefore we have proved that $\mu\left(M_{1} \backslash G_{M}\right)=0$. Similarly, we prove that $v\left(N_{1} \backslash\right.$ $\left.G_{N}\right)=0$.

If $p \in G_{M}$, we have that $\bigcap_{n=1}^{\infty} J_{n}^{-1}\left(I_{n}(p)\right)$ is a nonempty subset of $N_{1}$. From Lemma 4.1 we see that there exists a unique point $q \in N_{1}$ such that

$$
\bigcap_{n=1}^{\infty} J_{n}^{-1}\left(I_{n}(p)\right)=\{q\} .
$$

Set $\beta(p)=q$. In particular, we conclude that $\beta$ is a well-defined bijection between $G_{M}$ and $G_{N}$. We also have $\beta=J_{\infty}^{-1} \circ I_{\infty}$.

\section{LEMMA 4.3}

The map $\beta: G_{M} \rightarrow G_{N}$ satisfies the following conditions.

(1) $\quad \beta$ is a bijection, and both $\beta$ and $\beta^{-1}$ are measurable maps. Also, $\beta$ and $\beta^{-1}$ map sets of measure zero onto sets of measure zero.

The equality

$$
\frac{T(\psi)}{T(\phi)}(\beta)=\frac{\psi}{\phi}
$$

holds on $G_{M}$ for every $\phi, \psi \in A^{1}(M)$. Let $p \in G_{M}$, and let $q \in N_{1}$. If

$$
\frac{T(\psi)}{T(\phi)}(q)=\frac{\psi}{\phi}(p)
$$

holds for every $\phi, \psi \in A^{1}(M)$, then $q=\beta(p)$.

Let $q \in G_{N}$, and let $p \in M_{1}$. If

$$
\frac{T(\psi)}{T(\phi)}(q)=\frac{\psi}{\phi}(p)
$$

holds for every $\phi, \psi \in A^{1}(M)$, then $p=\beta^{-1}(q)$.

If $F$ is a measurable subset of $G_{M}$, then

$$
\int_{F}|\phi|=\int_{\beta(F)}|T(\phi)|
$$

holds for every $\phi \in A^{1}(M)$. In particular, we have $\mu(F)=v(\beta(F))$. 
Proof

It follows from Lemma 4.1 that $\beta$ is a bijection between $G_{M}$ and $G_{N}$. From the definition of $G_{M}, G_{N}, I_{n}, J_{n}$, and since $\phi_{n}$ is dense in $A^{1}(M)$, we see that

$$
\frac{T(\phi)}{T\left(\phi_{0}\right)}(\beta)=\frac{\phi}{\phi_{0}}
$$

on $G_{M}$. Since for $\phi, \psi \in A^{1}(M)$ we have

$$
\frac{\phi}{\phi_{0}} / \frac{\psi}{\phi_{0}}=\frac{\phi}{\psi}
$$

it follows that $\beta$ satisfies condition (2). The fact that $\beta$ satisfies conditions (3) and (4) follows directly from Lemma 4.1.

It is left to show that $\beta$ satisfies condition (5). Let $F$ be a compact set in $G_{M}$. Let $u_{n}: \mathbf{C}^{n} \rightarrow \mathbf{R}$ be the function given by

$$
u_{n}\left(w_{1}, \ldots, w_{n}\right)=\left|w_{1}\right|,
$$

where $\left(w_{1}, \ldots, w_{n}\right) \in \mathbf{C}^{n}$. Let $v_{n}$ be the function defined as the product of $u_{n}$ and the characteristic function of the set $I_{n}(F)$. From the proof of Lemma 2.2 applied to the function $v_{n}$, we find

$$
\int_{I_{n}^{-1}\left(I_{n}(F)\right)}\left|\phi_{1}\right| d x d y=\int_{J_{n}^{-1}\left(I_{n}(F)\right)}\left|T\left(\phi_{1}\right)\right| d x_{1} d y_{1} .
$$

In particular, since $\beta(F)$ is a Borel set, we have that the above integrals are well defined. Since $I_{n+1}^{-1}\left(I_{n+1}(F)\right)$ is contained in $I_{n}^{-1}\left(I_{n}(F)\right)$, from Lemma 4.1 we have that the set

$$
\bigcap_{n=1}^{\infty} I_{n}^{-1}\left(I_{n}(F)\right)
$$

is equal to $F$. Now, from Lemma 4.2 we conclude that

$$
\left|\int_{I_{n}^{-1}\left(I_{n}(F)\right)}\right| \phi_{1}\left|d x d y-\int_{F}\right| \phi_{1}|d x d y| \rightarrow 0, \quad n \rightarrow \infty .
$$

Similarly, we obtain

$$
\left|\int_{J_{n}^{-1}\left(I_{n}(F)\right)}\right| T\left(\phi_{1}\right)\left|d x d y-\int_{\beta(F)}\right| T\left(\phi_{1}\right)\left|d x_{1} d y_{1}\right| \rightarrow 0, \quad n \rightarrow \infty .
$$

So far we have verified (5) whenever $F$ is a compact subset of $G_{M}$. Applying the standard argument, we obtain that (5) holds for every measurable set $F$ contained in $G_{M}$. From (5) we also conclude that $\beta$ maps the sets of measure zero onto sets of measure zero. This proves claim (1). 
In the same way, we can show that (5) holds for every $\phi_{n}$, and from the density of the sequence $\left(\phi_{n}\right)$ we have that (5) holds for every $\phi \in A^{1}(M)$.

If we take $\phi=\phi_{0}$, we see that $\beta$ and $\beta^{-1}$ are measure-preserving maps; that is, the equality $\mu(F)=v(\beta(F))$ holds for every measurable set $F$ in $G_{M}$.

\section{Construction of the map $\alpha$, and its properties}

Define the sets $E_{M}$ and $E_{N}$ as follows.

Definition

We say that a point $p \in M$ belongs to $E_{M}$ if there exist an open set $U$ containing $p$ and the the holomorphic injection $\alpha: U \rightarrow N$, such that the equality

$$
\frac{T(\psi)}{T(\phi)} \circ \alpha=\frac{\psi}{\phi}
$$

holds on $U$ for every $\phi, \psi \in A^{1}$. In a similar way, we define the set $E_{N}$.

It follows that the set $E_{M}$ is an open subset of $M . E_{N}$ is an open subset of $N$. We show that the map $\alpha$ is a well-defined holomorphic bijection between $E_{M}$ and $E_{N}$.

To show that $\alpha$ is well defined, we need to consider two different open sets $U_{1}$ and $U_{2}$ and two holomorphic injections $\alpha: U_{1} \rightarrow N$ and $\alpha_{2}: U_{1} \rightarrow N$, which both satisfy (5.1), and to show that $\alpha_{1}$ and $\alpha_{2}$ agree on $U_{1} \cap U_{2}$. Combining the fact that $M \backslash M_{1}$ is a discrete set and items (3) and (4) from Lemma 4.3 together with (5.1), we conclude that $\alpha_{k}=\beta$ almost everywhere on $U_{k}$ for $k=1,2$. This implies that $\alpha_{1}$ and $\alpha_{2}$ agree almost everywhere on $U_{1} \cap U_{2}$. Similarly, we show that $\alpha$ is a bijection between $E_{M}$ and $E_{N}$.

\section{LEMMA 5.1}

We have the following.

(1) Let $p_{0} \in M_{1}$, and let $\left(p_{n}\right)_{n \in \mathbf{N}}$ be a sequence of points such that $p_{n} \in G_{M}$ and $p_{n} \rightarrow p_{0}$. If there is a point $q_{0} \in N_{1}$ such that $\beta\left(p_{n}\right) \rightarrow q_{0}$, then the points $p_{0}$ and $q_{0}$ belong to $E_{M}$ and $E_{N}$, respectively. The sets $E_{M}$ and $E_{N}$ have full measure in $M$ and $N$, respectively. The map $\alpha: E_{M} \rightarrow E_{N}$ is a holomorphic bijection between $E_{M}$ and $E_{N}$.

(3) We have that if $p \in E_{M} \cap M_{1}$ and $\alpha(p) \in N_{1}$, then $p \in G_{M}$. Similarly, if $q \in E_{N} \cap N_{1}$ and $\alpha^{-1}(q) \in M_{1}$, then $q \in G_{N}$. The maps $\alpha$ and $\beta$ agree almost everywhere on $M$. Also, $\alpha^{-1}$ and $\beta^{-1}$ agree almost everywhere on $N$.

$$
\left|(T(\phi) \circ \alpha)\left(\alpha^{\prime}\right)^{2}\right|=|\phi|
$$

holds on $E_{M}$ for every $\phi \in A(M)$. 
Proof

Since $p_{n} \in G_{M}$, from property (2) of Lemma 4.3, we conclude that

$$
\frac{T(\psi)}{T(\phi)}\left(\beta\left(p_{n}\right)\right)=\frac{\psi}{\phi}\left(p_{n}\right)
$$

for every $\psi, \phi \in A^{1}(M)$. Since $p_{n} \rightarrow p_{0} \in M_{1}$ and $\beta\left(p_{n}\right) \rightarrow q_{0} \in N_{1}$, we have

$$
\frac{T(\psi)}{T(\phi)}\left(q_{0}\right)=\frac{\psi}{\phi}\left(p_{0}\right)
$$

for every $\phi, \psi \in A^{1}(M)$. On the other hand, from the definition of $M_{1}$ and $N_{1}$, we know that there are open neighborhoods $U_{0}$ and $V_{0}$ of points $p_{0}$ and $q_{0}$, respectively, such that the functions $\left(\phi_{1} / \phi_{0}\right)(p)$ and $\left(T\left(\phi_{1}\right) / T\left(\phi_{0}\right)\right)(q)$ are univalent as maps from $U_{0}$ and $V_{0}$, respectively, into $\mathbf{C}$. By modifying the neighborhoods $U_{0}$ and $V_{0}$ if necessary, we see that there exists a conformal map $\tilde{\alpha}: U_{0} \rightarrow V_{0}$ such that the relation

$$
\frac{T\left(\phi_{1}\right)}{T\left(\phi_{0}\right)} \circ \tilde{\alpha}=\frac{\phi_{1}}{\phi_{0}}
$$

holds on $U_{0}$. Here we choose $U_{0}$ and $V_{0}$ small enough so that they belong to $M_{1}$ and $N_{1}$, respectively.

Since $\tilde{\alpha}$ is conformal on $U_{0}$, it follows from (5.2), and (5.3), that $\tilde{\alpha}\left(p_{n}\right)=\beta\left(p_{n}\right)$ for all but finitely many $n \in \mathbf{N}$. Also, the equality

$$
\frac{T(\psi)}{T(\phi)}\left(\tilde{\alpha}\left(p_{n}\right)\right)=\frac{\psi}{\phi}\left(p_{n}\right)
$$

holds for all but finitely many $n \in \mathbf{N}$ and for every $\phi, \psi \in A^{1}(M)$. Since $p_{n} \rightarrow p_{0}$, we conclude that

$$
\frac{T(\psi)}{T(\phi)}(\tilde{\alpha}(p))=\frac{\psi}{\phi}(p)
$$

for every $p \in U_{0}$ and for every $\phi, \psi \in A^{1}(M)$. From property (3) of Lemma 4.3 and from the above equality, together with the fact that $U_{0}$ is a subset of $M_{1}$ and $V_{0}$ is a subset of $N_{1}$, we conclude that $\beta$ is defined in $U_{0}$ and that $U_{0}$ is an open subset of $G_{M}$. This proves Lemma 5.1(1).

The map $\beta$ is a measurable map. In fact, if we ignore some sets of measure zero, we can think of the map $\beta$ as a map from the interior of a fundamental polygon for $M$ to the interior of such a polygon for $N$. This makes $\beta$ an $L^{1}$-function on a plane region, and we can talk about the set of Lebesgue points of the map $\beta$ on the interior of the fundamental polygon for $M$. The corresponding points on the surface $M$ are called the Lebesgue points for $\beta$. Note that this definition of the Lebesgue points on $M$ for $\beta$ depends on the choice of the fundamental polygon for $M$. Nevertheless, this set has full measure in $M$. 
Since $\beta$ maps sets of positive measure onto sets of positive measure, we conclude that for almost every Lebesgue point $p_{0} \in G_{M}$ the following holds. There exists a point $q_{0} \in G_{N}$ and a sequence of distinct points $\left(p_{n}\right)_{n \in \mathbf{N}}$, such that $p_{n} \in G_{M}$, $p_{n} \rightarrow p_{0}$, and $\beta\left(p_{n}\right) \rightarrow q_{0}$. Therefore every such Lebesgue point is contained in $E_{M}$, and we conclude that $E_{M}$ has full measure in $M$.

We have already shown that $\alpha$ is the well-defined holomorphic bijection between $E_{M}$ and $E_{N}$. Also, if $p \in E_{M} \cap M_{1}$ and $\alpha(p) \in N_{1}$, it follows from the definition of $G_{M}$ that $p \in G_{M}$. Similarly, we show the corresponding result for $E_{N} \cap N_{1}$. We have seen that $\alpha$ agrees with $\beta$ almost everywhere on any open subset of $E_{M}$. Since $E_{M}$ has full measure in $M$, we conclude that $\alpha$ and $\beta$ agree almost everywhere on $M$. In the same way, we show that $\alpha^{-1}$ agrees with $\beta^{-1}$ almost everywhere on $N$.

For every $\phi \in A^{1}(M)$, we define

$$
L(\phi)=(T(\phi) \circ \alpha)\left(\alpha^{\prime}\right)^{2} .
$$

The differential $L(\phi)$ is defined on $E_{M}$. Also, it follows from Lemma 4.3(5) that for every measurable set $F$ which is a subset of $E_{M}$, we have

$$
\int_{F}|\phi| d x d y=\int_{F}|L(\phi)| d x d y .
$$

Since $\phi$ and $L(\phi)$ are holomorphic forms on $E_{M}$, and $E_{M}$ is an open set in $M$, it follows from (5.4) that

$$
\left|(T(\phi) \circ \alpha)\left(\alpha^{\prime}\right)^{2}\right|=|\phi|
$$

holds everywhere on $E_{M}$ and for every $\phi \in A^{1}(M)$.

LEMMA 5.2

Let $p^{\prime} \in M$, and assume that there exists an open set $U$ which contains $p^{\prime}$ such that $U \backslash\left\{p^{\prime}\right\}$ is contained in $E_{M}$. Then $p^{\prime} \in E_{M}$.

Proof

Denote by $\tilde{\alpha}$ the extension of the map $\alpha$ on the set $U$. Since $U \backslash\left\{p^{\prime}\right\}$ is a subset of $E_{M}$, we have that $\tilde{\alpha}$ satisfies equality (5.1) on $U \backslash\left\{p^{\prime}\right\}$. If $\tilde{\alpha}\left(p^{\prime}\right)$ belongs to $N$, then $\tilde{\alpha}$ satisfies (5.1) on $U$ as well, and by the definition of $E_{M}$, we have $p^{\prime} \in E_{M}$. So, we may assume that $\tilde{\alpha}\left(p^{\prime}\right)$ is not in $N$. Then $\tilde{\alpha}\left(p^{\prime}\right)$ has to be a puncture in the boundary of $N$.

It is well known that there exists $\phi \in A(N)$ such that $\phi$ has a first-order pole at a given puncture in the boundary of $M$, in this case, the puncture $\tilde{\alpha}\left(p^{\prime}\right)$. From Lemma 5.1(4), we see that the equality

$$
\left|((\phi) \circ \tilde{\alpha})\left(\tilde{\alpha}^{\prime}\right)^{2}\right|=\left|T^{-1}(\phi)\right|
$$


holds on $U \backslash\left\{p^{\prime}\right\}$ and therefore on $U$ as well. Since $T^{-1}(\phi)$ is holomorphic at $p^{\prime}$, we obtain a contradiction.

Let us repeat that the sets $M \backslash M_{1}$ and $N \backslash N_{1}$ are discrete sets of points in $M$ and $N$, respectively. We have also shown that if $p^{\prime}$ is a puncture in the boundary of $M$, then $p^{\prime}$ is not a cluster point of the set $M \backslash M_{1}$. The same holds for punctures in the boundary of $N$.

LEMMA 5.3

The sets $M \backslash E_{M}$ and $N \backslash E_{N}$ have no isolated points. Furthermore, assume that $\left(p_{n}\right)_{n \in \mathbf{N}}$ is a sequence of distinct points such that every $n \in \mathbf{N}, p_{n} \in E_{M}$, and such that $p_{n} \rightarrow p_{0}$, where $p_{0} \in M_{1} \backslash E_{M}$. Then the sequence $\alpha\left(p_{n}\right)$ has no cluster points in $N_{1}$.

The same conclusion holds for the set $E_{N}$.

Proof

The fact that $M \backslash E_{M}$ and $N \backslash E_{N}$ have no isolated points follows directly from Lemma 5.2.

Assume that the sequence $\alpha\left(p_{n}\right)$ has a cluster point $q \in N_{1}$. By passing to a subsequence if necessary, we may assume $\alpha\left(p_{n}\right) \rightarrow q_{0}$, where $q_{0} \in N_{1}$. Since $p_{0} \in$ $M_{1}$, from Lemma 5.1(1) we conclude that $p_{0} \in E_{M}$, which is a contradiction. This proves the second part of this lemma.

\section{The boundary behavior of the map $\alpha$}

Let $\Gamma_{N}$ be the Fuchsian group acting on the unit disc $\Delta$ such that $\Delta / \Gamma_{N}$ is conformally equivalent to the surface $N$. Denote by $P: \Delta \rightarrow N$ the universal covering map.

Let $\left(z_{n}\right)_{n \in \mathbf{N}}$ be a sequence of distinct points in $\Delta$ such that

(1) $\left|z_{n}\right| \rightarrow 1, n \rightarrow \infty$;

(2) there exists a sequence $r_{n}>1$ such that $r_{n} \rightarrow \infty$ for $n \rightarrow \infty$, and the covering map $P: \Delta \rightarrow N$ is univalent in the hyperbolic disc of radius $r_{n}$, centered at $z_{n}$

(3) if by $q_{n}$ we denote the points on $N$ such that $q_{n}=P\left(z_{n}\right)$, then there exists a sequence of distinct points $\left(p_{n}\right), p_{n} \in E_{M} \cap M_{1}$, for every $n \in \mathbf{N}$, such that $\alpha\left(p_{n}\right)=q_{n}$.

Our aim in this section is to show that under the stated assumptions the sequence $p_{n}$ cannot have a cluster point in $M_{1}$.

If $M$ has a positive genus, we have that the space $\Gamma(M)$ of Abelian differentials of finite norm is of positive dimension. From Virtanen's theorem (see [10, page 43]), 
it follows that for every point $p \in M$ there exists $\varphi \in \Gamma(M)$ such that $\varphi(p) \neq 0$. Then $(\varphi)^{2}(p) \neq 0$. We conclude that for every point $p \in M$, there exists $\phi \in A^{1}(M)$ such that $\phi(p) \neq 0$.

If $M$ is a planar region, then using the fact that $M$ is not of exceptional type, for every point $p \in M$, we can construct a rational differential of $M$ which does not have zero at $p$.

For each $p_{n}$, denote by $Z_{n}(M)$ the space of all $\phi \in A^{1}(M)$ such that $\phi\left(p_{n}\right)=$ 0 . Also, for every $q_{n}$ we denote by $Z_{n}(N)$ the space corresponding to $q_{n}$. We see that $Z_{n}(M)$ is a vector subspace of $A^{1}(M)$ of codimension 1. Repeating the same argument, we conclude that $Z_{n}(N)$ is a vector subspace of $A^{1}(N)$ of codimension 1.

We have $\alpha\left(p_{n}\right)=q_{n}$. Since $\phi_{0} \neq 0$ on $M_{1}$ ( $\phi_{0}$ was determined at the beginning of Section 4.), from (5.1) we conclude that $T\left(Z_{n}(M)\right)=Z_{n}(N)$. Let $\lambda_{n}: A^{1}(M) \rightarrow \mathbf{C}$ be a linear functional of the Banach norm equal to 1 which annihilates the subspace $Z_{n}(M)$; that is, $\lambda_{n}\left(Z_{n}(M)\right)=0$. Let $\lambda_{n}^{\prime}: A^{1}(N) \rightarrow \mathbf{C}$ be the linear functional such that $\lambda_{n}(\phi)=\lambda_{n}^{\prime}(T(\phi))$ for every $\phi \in A^{1}(M)$. Since $T$ is a linear isometry, we have that $\lambda_{n}^{\prime}$ is of norm 1 , and by definition, $\lambda^{\prime}$ annihilates the subspace $Z_{n}(N)$ of $A^{1}(N)$.

We now show that from the assumptions on the sequence $z_{n}$ and the group $\Gamma_{N}$, it follows that for every $\phi \in A^{1}(N)$ we have $\lambda_{n}^{\prime}(\phi) \rightarrow 0$.

From assumption (2) it follows that the covering map $P$ is univalent on the hyperbolic disc $\mathbf{D}_{n}$ centered at $z_{n}$, and has hyperbolic radius 1 . Let $B_{n}$ be a Möbius transformation of the unit disc onto itself such that $B_{n}\left(z_{n}\right)=0$. Define the function $\eta_{n}$ to be equal to $\overline{\left(B_{n}^{\prime}\right)} / B_{n}^{\prime}$ on $\mathbf{D}_{n}$. For $A \in \Gamma_{N}$, set $\eta_{n}$ to be equal to

$$
\left(\frac{\overline{\left(B_{n}^{\prime}\right)}}{B_{n}^{\prime}} \circ A\right) \frac{\overline{\left(A^{\prime}\right)}}{A^{\prime}}
$$

on the disc $A^{-1}\left(\mathbf{D}_{n}\right)$. Elsewhere in the unit disc, we define $\eta_{n}$ to be equal to zero.

Denote by $A^{1}(\Delta)$ the Banach space of all integrable analytic functions on the unit disc. Also, denote by $\Theta$ the standard Poincaré operator $\Theta: A^{1}(\Delta) \rightarrow A^{1}(N)$. The operator $\Theta$ is surjective, and its Banach norm is at most 1 . We have that the covering map $P$ is univalent on $\mathbf{D}_{n}$, and since $\eta_{n}$ is equivariant, we see that $\eta_{n}$ represents the lift of a certain $(-1,1)$ measurable form on $N$.

Let $\omega \in A^{1}(\Delta)$. From the mean value theorem for analytic functions, we obtain

$$
\int_{\Delta} \eta_{n} \omega=d \sum_{A \in \Gamma_{N}}(\omega \circ A)\left(A^{\prime}\right)^{2}\left(z_{n}\right)
$$

where $d>0$ is some constant. Therefore we have

$$
\int_{\Delta} \eta_{n} \omega=0
$$

if and only if $\Theta(\omega) \in Z_{n}(N), \omega \in A^{1}(\Delta)$. 
Therefore there exists $t_{n} \in \mathbf{C}$ such that for each $\omega \in A^{1}(\Delta)$ we have

$$
\int_{\Delta} t_{n} \eta_{n} \omega=\lambda_{n}^{\prime}(\Theta(\omega))
$$

Set $\omega_{n}=\left(B_{n}^{\prime}\right)^{2}$. We have that the Banach norm $\left\|\omega_{n}\right\|_{1}$ is equal to $\pi$. Also, from the properties of the sequence $z_{n}$, it follows that

$$
\int_{\Delta} \eta_{n} \omega_{n}=\int_{D_{n}} \eta_{n} \omega_{n}+\sum_{A \in\left(\Gamma_{N} \backslash \mathrm{Id}\right)} \int_{A\left(D_{n}\right)} \eta_{n} \omega_{n} .
$$

From the properties of the sequence $z_{n}$, it follows that as $n \rightarrow \infty$ the hyperbolic distance between $z_{n}$ and any point $A\left(z_{n}\right), A \in\left(\Gamma_{N} \backslash \mathrm{Id}\right)$, uniformly tends to $\infty$. From the construction of the function $\omega_{n}$, it follows that

$$
\sum_{A \in\left(\Gamma_{N} \backslash \mathrm{Id}\right)} \int_{A\left(D_{n}\right)}\left|\omega_{n}\right| \rightarrow 0,
$$

and we have

$$
\left|\int_{\Delta} \eta_{n} \omega_{n}\right| \rightarrow c_{1}
$$

when $n \rightarrow \infty$, and $z_{n} \rightarrow x$. Here, $c_{1}$ stands for the Euclidean area of the hyperbolic disc of the hyperbolic radius 1 , centered at the origin in the unit disc.

We have constructed the sequence $\varphi_{n}=\Theta\left(\omega_{n}\right) \in A^{1}(N)$ so that $\left\|\varphi_{n}\right\|_{1} \leq \pi$ and

$$
\lim _{n \rightarrow \infty} \frac{\left|\lambda^{\prime}\left(\varphi_{n}\right)\right|}{\left|t_{n}\right|}=c_{1}
$$

Since $\left|\lambda^{\prime}\left(\varphi_{n}\right)\right| \leq \pi$, we conclude that $\lim \sup \left(\left|t_{n}\right|\right) \leq \pi / c_{1}$.

We have that $t_{n} \eta_{n}$ is uniformly bounded on the unit disc (the bound does not depend on $n$ ), and we have that $t_{n} \eta_{n}$ converges to zero pointwise on the unit disc. This conclusion, together with (6.1), yields that for any fixed $\omega \in A^{1}(\mathbf{D})$, we have

$$
\int_{\Delta} t_{n} \eta_{n} \omega=\lambda_{n}^{\prime}(\Theta(\omega)) \rightarrow 0, \quad n \rightarrow \infty .
$$
$A^{1}(N)$.

Since $\Theta$ is surjective, we have shown that $\lambda_{n}^{\prime}(\phi) \rightarrow 0, n \rightarrow \infty$, for every $\phi \in$

We have the following.

LEMMA 6.1

With the notation and assumptions stated above, we have that the sequence $p_{n}$ has no cluster points in $M_{1}$. 
Proof

Assume now that the sequence $p_{n}$ has a cluster point in $M_{1}$. By passing onto a subsequence if necessary, we may assume that there exists a point $p_{0} \in M_{1}$ such that $p_{n} \rightarrow p_{0}$. Let $\psi_{0} \in A^{1}(M)$ such that $\psi_{0}\left(p_{0}\right) \neq 0$. We show that the sequence $\lambda_{n}\left(\psi_{0}\right)$ does not converge to zero.

To see that $\lambda_{n}\left(\psi_{0}\right)$ does not converge to zero, note that for $n$ large enough the functional $\lambda_{n}$ is a multiple of the linear functional

$$
\psi \rightarrow \frac{\psi}{\psi_{0}}\left(p_{n}\right), \quad \psi \in A^{1}(M) .
$$

Since the above functional converges to the linear functional

$$
\psi \rightarrow \frac{\psi}{\psi_{0}}\left(p_{0}\right), \quad \psi \in A^{1}(M),
$$

which is a nonzero linear functional, we see that the sequence $\lambda_{n}\left(\psi_{0}\right)$ does not converge to zero.

\section{Connected components of the set $E_{M}$}

Let $p_{0} \in E_{M}$, and let $E_{M, 0}$ be the maximal connected component of the set $E_{M}$ which contains $p_{0}$. That is, the set $E_{M, 0}$ is the union of all connected components of $E_{M}$ containing the point $p_{0}$.

\section{LEMMA 7.1}

Let $\gamma$ be a Jordan curve in $E_{M, 0}$ such that the Jordan domain $\Omega_{0}$ bounded by $\gamma$ is a well-defined planar subregion of $M_{1}$ and such that $p_{0} \in \Omega_{0}$. Then $\Omega_{0}$ is contained in $E_{M, 0}$.

Proof

Set $D_{0}=\Omega_{0} \cap E_{M, 0}$. Our aim is to show $D_{0}=\Omega_{0}$. Since $\gamma$ is contained in $E_{M, 0}$, we see that $\gamma$ is an isolated boundary component of $D_{0}$. Also, set $\tilde{D}_{0}=\alpha\left(D_{0}\right)$. As a Riemann surface, the region $\tilde{D}_{0}$ is embedded in the surface $N$. We see that $\alpha(\gamma)$ is an isolated component of the boundary of the region $\tilde{D}_{0}$ in $N$. We now show that this is the only component of the boundary $\partial{\tilde{D_{0}}}_{0}$ in $N$. This means that the region $\tilde{D}_{0}$ is a well-defined $e n d$ of $N$ in the sense of [10, page 3]. Let $B$ be a component of the boundary $\partial \tilde{D}_{0}$ in $N$. In particular, $B$ is a closed set in $N$. If the component $B$ is different from $\alpha(\gamma)$, we conclude that $B \cap E_{N}$ is an empty set. Choose an arbitrary point $q^{\prime} \in B$. Let $\left(q_{n}\right)_{n \in \mathbf{N}}$ be a sequence of distinct points in $\tilde{D}_{0}$ such that $q_{n} \rightarrow q^{\prime}$. Let $p^{\prime}$ be a cluster point of the sequence $\alpha^{-1}\left(q_{n}\right)$. Then $p^{\prime}$ belongs to the closure of the set $D_{0}$. Since $\alpha$ is conformal and $B$ is a boundary component different from $\alpha(\gamma)$, $p^{\prime}$ cannot belong to the curve $\gamma$. 
Assume now that $q^{\prime} \in N_{1}$. Since $q_{n} \rightarrow q^{\prime}, q_{n} \in E_{N}$, and $q^{\prime} \in N$, from Lemma 5.3 we conclude that the sequence $\alpha^{-1}\left(q_{n}\right)$ has no cluster points in $M_{1}$. But this is not possible since $p^{\prime}$ does not belong to $\gamma$, and the closure of $D_{0}$ is contained in $M_{1}$.

So, the only possibility left is that $q^{\prime} \in N \backslash N_{1}$. Therefore we have that the whole component $B$ has to be a subset of $N \backslash N_{1}$. But $B$ is connected, so it has to reduce to a single point $q^{\prime}$. Then $q^{\prime}$ is an isolated point in the set $N \backslash E_{N}$, which is not possible by Lemma 5.3.

Therefore we have proved that ${\tilde{D_{0}}}_{0}$ is a regularly embedded region in $N$; that is, $\tilde{D}_{0}$ is an end in $N$. The Jordan curve $\alpha(\gamma)$ is the boundary of $\tilde{D}_{0}$. Also, since $D_{0}$ is a planar region, we conclude that $\alpha\left(D_{0}\right)=\tilde{D}_{0}$ (as a Riemann surface) is a planar region too. In other words, $\tilde{D}_{0}$ is a planar end in $N$. Then, it is well known (see [10, page 258 and Theorem 5A]) that there exist a Riemann surface $N^{*}$ and a compact set $S$ in $N^{*}$, such that

(1) $N^{*} \backslash S=N$;

(2) $\tilde{D}_{0} \cup S=\Omega_{0}^{*}$ is a Jordan region bounded by $\alpha(\gamma)$.

We also define the set $E$ to be the boundary of the set $S$ in $N^{*}$.

Remark. It is elementary to construct the surface $N^{*}$ (see [10, page 258]). The surface $N^{*}$ is called an extension of the surface $N$.

By construction, there exists a Jordan region $\Omega_{0}^{*}$ which is a subset of $N^{*}$ and which is bounded by the Jordan curve $\alpha(\gamma)$. But in general this by no means suggests that the map $\alpha$ can be conformally extended to map $\Omega_{0}$ onto $\Omega_{0}^{*}$. But in our case this is true, and it is a corollary of the existence of the linear isometry $T: A^{1}(M) \rightarrow A^{1}(N)$. However, we do not have to go that far in order to prove this lemma.

If the set $E$ defined above is not empty, then it cannot consist of finitely many points. If $E$ consisted of finitely many points, then the map $\alpha^{-1}$ could be extended over $E$, and map $E$ into $M \backslash E_{M}$. But every point in $\alpha^{-1}(E)$ is then an isolated point of the set $M \backslash E_{M}$, which is not possible by Lemma 5.3.

Since $E$ contains infinitely many points, we can choose a finite set $E^{\prime}$ which is a subset of $E$, and such that $E^{\prime}$ contains at least five points. Then, we can choose two linearly independent $\tilde{\phi}, \tilde{\psi} \in A^{1}\left(N^{*} \backslash E^{\prime}\right)$. The function $\tilde{\psi} / \tilde{\phi}$ is a well-defined meromorphic function on $N^{*} \backslash E^{\prime}$, and in particular, there are only finitely many points in $\Omega_{0}^{*}$ in which the function $\tilde{\psi} / \tilde{\phi}$ has poles or its first derivative is equal to zero. Since $\tilde{\phi}, \tilde{\psi} \in A^{1}\left(N^{*} \backslash E^{\prime}\right)$ and $E^{\prime}$ is a subset of $E$, we have $\tilde{\phi}, \tilde{\psi} \in A^{1}(N)$. (It is understood that the restrictions of $\tilde{\phi}, \tilde{\psi}$ on $N$ are in $A^{1}(N)$.) The function $T^{-1}(\tilde{\psi}) / T^{-1}(\tilde{\phi})$ is a well-defined meromorphic function on $M$, and in particular, there are only finitely many points $\Omega_{0}$ in which the function $T^{-1}(\tilde{\psi}) / T^{-1}(\tilde{\phi})$ has poles or its first derivative 
is equal to zero. From Lemma 5.1 we have that the equality

$$
\frac{T^{-1}(\tilde{\psi})}{T^{-1}(\tilde{\phi})}=\frac{\tilde{\psi}}{\tilde{\phi}} \circ \alpha
$$

holds on $D_{0}$.

Since $\alpha$ is a conformal map between $D_{0}$ and $\tilde{D}_{0}$, we see that one can choose points $p$ in the closure of $D_{0}$ (and $p$ is not in $\gamma$ ) and $q \in\left(E \backslash E^{\prime}\right)$, such that the following holds. There exists a sequence of distinct points $\left(q_{n}\right)_{n \in \mathbf{N}}, q_{n} \in \tilde{D}_{0}$, such that $q_{n} \rightarrow q$ and $\alpha^{-1}\left(q_{n}\right) \rightarrow p$. Also, we can arrange that the functions $T^{-1}(\tilde{\psi}) / T^{-1}(\tilde{\phi})$, and $\tilde{\psi} / \tilde{\phi}$ are univalent and bounded in some (sufficiently small) neighborhoods of the points $p$ and $q$, respectively. Denote these neighborhoods by $U$ and $V$, respectively. Since $q_{n} \in E_{N}$, and from (7.1), it follows that

$$
\frac{T^{-1}(\tilde{\psi})}{T^{-1}(\tilde{\phi})}(p)=\frac{\tilde{\psi}}{\tilde{\phi}}(q)
$$

Therefore, by modifying $U$ and $V$ if necessary, we see that there exists a conformal map $\tilde{\alpha}: U \rightarrow V$ such that the equality

$$
\frac{T^{-1}(\tilde{\psi})}{T^{-1}(\tilde{\phi})}=\frac{\tilde{\psi}}{\tilde{\phi}} \circ \tilde{\alpha}
$$

holds on $U$. Set $U^{\prime}=\alpha^{-1}\left(V \cap \tilde{D_{0}}\right) \cap U$. Since $q_{n} \rightarrow q$ and $\alpha^{-1}\left(q_{n}\right) \rightarrow p$, we see that $U^{\prime}$ is a nonempty open set. Then, from (7.1) and (7.2), and since both $\alpha$ and $\tilde{\alpha}$ are injections, we conclude that $\alpha$ and $\tilde{\alpha}$ agree on $U^{\prime}$. From Lemma 5.1(4) we see that the equality

$$
\left|(\phi \circ \tilde{\alpha})\left(\tilde{\alpha}^{\prime}\right)^{2}\right|=\left|T^{-1}(\phi)\right|
$$

holds on $U^{\prime}$ for every $\phi \in A^{1}(N)$.

We now choose $\varphi \in A^{1}\left(N^{*} \backslash\left(E^{\prime} \cup\{q\}\right)\right)$ such that $\varphi$ has the first-order pole at $q$. From (7.3) it follows that

$$
\left|(\varphi \circ \tilde{\alpha})\left(\tilde{\alpha}^{\prime}\right)^{2}\right|=\left|T^{-1}(\varphi)\right|
$$

in $U \backslash\{g\}$. Therefore $T^{-1}(\varphi)$ has the first-order pole at $p$. Since $T^{-1}(\varphi) \in A^{1}(M)$, we have that $T^{-1}(\varphi)$ is holomorphic at $p$, which is a contradiction.

So, we have proved that the set $E$ is an empty set, and therefore it follows that $\Omega_{0}=D_{0}$. This proves Lemma 7.1.

\section{Proof of Theorem 1.1}

In this section we show that $E_{M}=M, E_{N}=N$ and that $\alpha$ is a conformal map between $M$ and $N$. 
Let $p_{0} \in E_{M}$, and as before, let $E_{M, 0}$ be the maximal connected component of the set $E_{M}$ which contains $p_{0}$. Also, let $\mathbf{D}_{0}$ be a hyperbolic disc centered at $p_{0}$ which is contained in a larger concentric hyperbolic disc which is injectively embedded in $M_{1}$. Set $\Omega=\mathbf{D}_{0} \cap E_{M, 0}$, and denote by $\Omega_{0}$ the connected component of the set $\Omega$ which contains $p_{0}$. We have that $\Omega_{0}$ is an open connected subset of $\mathbf{D}_{0}$.

LEMMA 8.1

We have $\mathbf{D}_{0}=\Omega_{0}$.

Proof

We assume that $\mathbf{D}_{0} \neq \Omega_{0}$.

It follows from Lemma 7.1 that $\Omega_{0}$ is a simply connected region. We define the following partition of the boundary $\partial \Omega_{0}$. Set $C=E_{M} \cap \partial \Omega_{0}$, and set $B=\partial \Omega_{0} \backslash C$. From the construction, we have that $C$ is an open subset of the circle $\partial \mathbf{D}_{0}$. Therefore $B$ is a compact set in $M$.

We recall the following statement proved in Section 5. Suppose that a point $q \in N$ belongs to the boundary of $\alpha\left(\Omega_{0}\right)$. If, in addition, we assume that $q$ does not belong to $E_{N}$, then from Lemma 5.3 we conclude that $q \in N \backslash N_{1}$. Therefore we have shown that if $\left(p_{n}\right)_{n \in \mathbf{N}}$ is a sequence of points in $\Omega_{0}$ such that $p_{n} \rightarrow B$, then the sequence $\alpha\left(p_{n}\right)$ has no cluster points in $N_{1}$. In particular, the only possible accumulation points (in $N$ ) of the sequence $\alpha\left(p_{n}\right)$ belong to the set $N \backslash N_{1}$.

Let $f: \Delta \rightarrow \Omega_{0}$ be a Riemann map with $f(0)=p_{0}$. Let $C_{1}$ be the open subset of the unit circle $\partial \Delta$ such that the map $f$ extends analytically onto $C_{1}$, and let $f: C_{1} \rightarrow C$. Denote by $B_{1}$ the complement of of the set $C_{1}$ with respect to the unit circle.

First, we study the case when the 1-dimensional Lebesgue measure of the set $B_{1}$ is zero. We may assume that $\mathbf{D}_{0}$ is in the complex plane. (Take a lift of $\mathbf{D}_{0}$ in the universal cover of $M$.) Since the measure of $B_{1}$ is zero, we have that $C_{1}$ is dense in $\partial \Delta$, and $B_{1}$ is a totally disconnected subset of the unit circle. But then the Riemann map $f: \Delta \rightarrow \Omega_{0}, f(0)=p_{0}$, can be conformally extended (by the reflection principle) onto $\mathbf{C} \backslash B_{1}$. Now, the extended map $f: \mathbf{C} \backslash B_{1} \rightarrow \mathbf{C}$ maps the region $\mathbf{C} \backslash B_{1}$ into the region $\mathbf{C} \backslash B$. Since $B_{1}$ is of measure zero, it follows (see [1]) that the extended map $f$ is a Möbius transformation and that $f(\Delta)=\mathbf{D}_{0}$, which is a contradiction.

Next, we obtain a contradiction to the assumption that $B_{1}$ has a positive measure. Set $\tilde{g}=\alpha \circ f$. Since $\alpha$ maps the domain $\Omega_{0}$ into $N_{1}$, we have that the map $\tilde{g}: \Delta \rightarrow N_{1}$ is a holomorphic injection. Denote by $g_{1}: \Delta \rightarrow \Delta$ the lift of the map $\tilde{g}$ into the universal cover (the unit disc) of the surface $N_{1}$ (note here that the points in $N \backslash N_{1}$ are isolated points in the boundary of $\left.N_{1}\right)$. Let $\left(z_{n}\right)_{n \in \mathbf{N}}$ be any sequence in the unit disc 
$\Delta$ such that $z_{n}$ converges to the set $B_{1}$. Then, as we have already pointed out, from Lemma 5.3 it follows that $\alpha\left(f\left(z_{n}\right)\right)$ does not have cluster points in $N_{1}$. This implies that $\left|g_{1}\left(z_{n}\right)\right| \rightarrow 1, n \rightarrow \infty$. This last fact, together with the fact that the measure of $B_{1}$ is positive, implies (see [6, Corollary 6.20]) that the map $g_{1}$ is conformal at almost every point of the set $B_{1}$ (see [6] for the notion of being conformal at boundary points). Let $X_{1}$ be the subset of $B_{1}$ whose elements are the points where the map $g_{1}$ is conformal. We just saw that $X_{1}$ has full measure in $B_{1}$.

Let $x \in X_{1}$. From [6, Proposition 4.10] we have that smooth curves in a Stolz angle at $x$ are mapped onto smooth curves, and the angles between curves are preserved. In particular, for every sequence $z_{n} \in \Delta$ such that $z_{n} \rightarrow x$ and $z_{n}$ is contained in a given Stolz angle at $x$, we have $r_{n} \rightarrow \infty$. Here $r_{n}$ is the radius of the maximal hyperbolic disc centered at $g_{1}\left(z_{n}\right)$ which is contained in $g_{1}(\Delta)$. Let $P_{1}: \Delta \rightarrow N_{1}$ be the corresponding universal covering map. Then, we conclude that the sequence $P_{1}\left(g_{1}\left(z_{n}\right)\right)$ cannot accumulate at any isolated puncture in the boundary of $N_{1}$. (This includes the points of the set $N \backslash N_{1}$.) This follows from the fact that if $q_{n}$ is a sequence on a given Riemann surface which converges to a puncture in the boundary of that surface, then the injectivity radius at $q_{n}$ goes to zero.

Denote by $g: \Delta \rightarrow \Delta$ the lift of the map $\tilde{g}$ into the universal cover (the unit disc) of the surface $N$, and let $P: \Delta \rightarrow N$ be the corresponding universal covering map. (Note here that the points in $N \backslash N_{1}$ are not in the boundary of $N$.) Let $x \in X_{1}$, and let $z_{n}$ be a sequence in $\Delta, z_{n} \rightarrow x$, and $z_{n}$ is contained in a given Stolz angle at $x$. Since we have that the corresponding sequence $P\left(g\left(z_{n}\right)\right)=P_{1}\left(g_{1}\left(z_{n}\right)\right)$ does not accumulate at any point in the set $N \backslash N_{1}$, and by using Lemma 5.3 again, we find that $\left|g\left(z_{n}\right)\right| \rightarrow 1$. This means that if the function $g$ has a radial limit at some point $x \in X_{1}$, then this limit lies on the unit circle.

On the other hand, the map $g$ has radial limits almost everywhere on the unit circle. Since $X_{1}$ is of positive measure and all the radial limits at points from $X_{1}$ lie on the unit circle, from [6, Corollary 6.20] we conclude that the map $g$ is conformal almost everywhere on $X_{1}$. Denote by $X$ the subset of $X_{1}$ which consists of points where $g$ is conformal. We just saw that $X$ has the full measure in $X_{1}$.

Now, we again apply [6, Proposition 4.10]. In a fashion similar to that above, we have that for every $x \in X$ and for every sequence $z_{n} \in \Delta, z_{n} \rightarrow x$, where $z_{n}$ is contained in a given Stolz angle at $x$, we have $r_{n} \rightarrow \infty$. Here $r_{n}$ is the radius of the maximal hyperbolic disc which is centered at $g\left(z_{n}\right)$ and contained in $g(\Delta)$. But the sequence $\alpha^{-1}\left(P\left(g\left(z_{n}\right)\right)\right)$ remains in the disc $\mathbf{D}_{0}$ which is compactly contained in $M_{1}$. From Lemma 6.1 we obtain a contradiction.

Therefore we have proved that $\Omega_{0}=\mathbf{D}_{0}$.

Lemma 8.1 implies that every injectively embedded hyperbolic disc in $M_{1}$ belongs 
to $E_{M}$. Therefore we have that $M_{1}$ is contained in $E_{M}$. By Lemma 5.3, there are no isolated points in $E_{M}$, so we conclude that $E_{M}=M$ and, similarly, that $E_{N}=N$. Therefore $\alpha: M \rightarrow N$ is a conformal map that by Lemma 5.1 satisfies the equality

$$
\left|(T(\phi) \circ \alpha)\left(\alpha^{\prime}\right)^{2}\right|=|\phi|
$$

for every $\phi \in A^{1}(M)$. Therefore for every $\phi \in A^{1}(M)$ there exists a complex number $\theta,|\theta|=1$ such that the equality

$$
(T(\phi) \circ \alpha)\left(\alpha^{\prime}\right)^{2}=\theta \phi
$$

holds on $M$. Since $T$ is a linear map, we find that the corresponding $\theta$ is the same for all $\phi \in A^{1}(M)$. This proves Theorem 1.1.

\section{The proofs of Theorems 1.2 and $\mathbf{1 . 3}$}

Both theorems are direct corollaries of Theorem 1.1 and the following propositions proved in [2].

\section{PROPOSITION 9.1}

Let $M$ and $N$ be two quasiconformally related Riemann surfaces that are not of exceptional type. If $M$ has the isometry property, then every biholomorphic map between $\operatorname{Teich}(M)$ and Teich $(N)$ is geometric.

\section{Proof of Theorem 1.2}

The above proposition shows that if Problem 1.1 has a positive answer for a Riemann surface $M$, then Conjecture 1.1 holds for $M$. Since Theorem 1.1 gives a positive answer to Problem 1.1, the proof of Theorem 1.2 follows from Proposition 9.1.

Teich $(M)$ is a complex Banach manifold. The tangent space at the origin of Teich $(M)$ is isometric to the Banach dual of the space $A^{1}(M)$. The following was proved in [2].

\section{PROPOSITION 9.2}

Suppose that $M$ and $N$ are not of exceptional type. If $F: \operatorname{Teich}(M) \rightarrow \operatorname{Teich}(N)$ is a biholomorphic map, then there exist Riemann surfaces $M^{\prime}$ and $N^{\prime}$ which are quasiconformally related to $M$ and $N$, respectively, and there exists a linear isometry $T: A^{1}\left(M^{\prime}\right) \rightarrow A^{1}\left(N^{\prime}\right)$.

\section{Proof of Theorem 1.3}

Let $F: \operatorname{Teich}(M) \rightarrow \operatorname{Teich}(N)$ be a biholomorphic map. If $M$ and $N$ are not of exceptional type, we need to show that $F$ is geometric.

First, we show that the existence of the map $F$ implies that $M$ and $N$ are quasiconformally related. We apply Proposition 9.2. Then from Theorem 1.1 we have 
that the linear isometry $T: A^{1}\left(M^{\prime}\right) \rightarrow A^{1}\left(N^{\prime}\right)$ is geometric, and therefore, by the definition in Section 1, $M$ and $N$ are conformally related. But this implies that $M$ and $N$ are quasiconformally related.

Now from Proposition 9.1 we conclude that $F$ is geometric. This proves Theorem 1.3.

Acknowledgments. I would like to thank the referee for a number of valuable comments and suggestions. In particular, I am grateful to the referee for pointing out to me that Lemma 3.2 holds for all Riemann surfaces of nonexceptional type. Also, the previous version of this paper contained the proof of Theorem 2.1. I am thankful to the referee for bringing to my attention the paper of Rudin [8], where this theorem was originally proved.

\section{References}

[1] L. V. AHLFORS AND L. SARIO, Riemann Surfaces, Princeton Math. Ser. 26, Princeton Univ. Press, Princeton, 1960. MR 22:5729

[2] C. J. EARLE and F. P. GARDINER, Geometric isomorphisms between infinite-dimensional Teichmüller spaces, Trans. Amer. Math. Soc. 348 (1996), 1163 - 1190. MR 96h:32024

[3] C. J. EARLE and I. KRA, On isometries between Teichmüller spaces, Duke Math. J. 41 (1974), 583-591. MR 50:596

[4] F. P. GARDINER, Teichmüller Theory and Quadratic Differentials, Pure Appl. Math., Wiley, New York, 1987. MR 88m:32044

[5] N. LAKIC, An isometry theorem for quadratic differentials on Riemann surfaces of finite genus, Trans. Amer. Math. Soc. 349 (1997), 2951 -2967. MR 97i:30062

[6] C. POMMERENKE, Boundary Behaviour of Conformal Maps, Grundlehren Math. Wiss. 299, Springer, Berlin, 1992. MR 95b:30008

[7] H. L. ROYDEN, "Automorphisms and isometries of Teichmüller space" in Advances in the Theory of Riemann Surfaces (Stony Brook, N.Y., 1969), Ann. of Math. Stud. 66, Princeton Univ. Press, Princeton, 1971, 369-383. MR 44:5452

[8] W. RUDIN, $L^{p}$-isometries and equimeasurability, Indiana Univ. Math. J. 25 (1976), 215-228. MR 53:14105

[9] - Function Theory in the Unit Ball of $\mathbf{C}^{n}$, Grundlehren Math. Wiss. 241, Springer, New York, 1980. MR 82i:32002

[10] L. SARIO and K. OIKAWA, Capacity Functions, Grundlehren Math. Wiss. 149, Springer, New York, 1969. MR 40:7441

[11] E. M. STEIN and G. WEISS, Fourier Analysis on Euclidean Spaces, Princeton Math. Ser. 32, Princeton Univ. Press, Princeton, 1971. MR 46:4102

Mathematics Institute, University of Warwick, Coventry CV4 7AL, United Kingdom; markovic@maths.warwick.ac.uk 